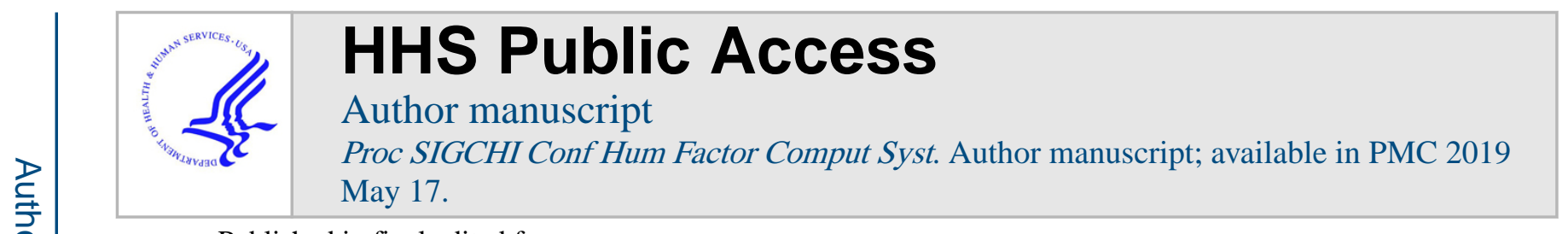

Published in final edited form as:

Proc SIGCHI Conf Hum Factor Comput Syst. 2019 May ; 2019: . doi:10.1145/3290605.3300518.

\title{
A Place to Play:
}

The (Dis)Abled Embodied Experience for Autistic Children in Online Spaces

\author{
Kathryn E. Ringland \\ Department of Communication Studies, Northwestern University, Chicago, IL, USA
}

\begin{abstract}
Play is the work of children-but access to play is not equal from child to child. Having access to a place to play is a challenge for marginalized children, such as children with disabilities. For autistic children, playing with other children in the physical world may be uncomfortable or even painful. Yet, having practice in the social skills play provides is essential for childhood development. In this ethnographic work, I explore how one community uses the sense of place and the digital embodied experience in a virtual world specifically to give autistic children access to play with their peers. The contribution of this work is twofold. First, I demonstrate how various physical and virtual spaces work together to make play possible. Second, I demonstrate these spaces, though some of them are digital, are no more or less "real" than the physical spaces making up a schoolyard or playground.
\end{abstract}

\section{Keywords}

Disability; Inclusion; Autism; Communication; Social Interaction; Virtual Worlds; Social Media; Embodied Experience

\section{INTRODUCTION}

Play is the work of children-but access to play is not equal from child to child. This is true for children with disabilities, including autistic children ${ }^{1}$, where playing with other children in the physical world may be uncomfortable or painful [51]. Yet, having practice social skills play provides is essential for childhood development [26].

One way to help autistic children gain access to play and socialization is through online spaces, such as social media and virtual worlds [9,10,59]. This paper extends previous work in this area, by exploring the disabled embodied experience and how that affects access to play. Access, in general, is not a given experience for any one person. When access is faulty or denied, disability is created in that moment [20]. Disability "is not simply lodged in the

Permission to make digital or hard copies of all or part of this work for personal or class-room use is granted without fee provided that copies are not made or distributed for profit or commercial advantage and that copies bear this notice and the full citation on the first page. Copyrights for components of this work owned by others than ACM must be honored. Abstracting with credit is permitted. To copy otherwise, or republish, to post on servers or to redistribute to lists, requires prior specific permission and/or a fee. Request permissions from permissions@acm.org).Publication rights licensed to ACM.

kathrynringland@northwestern.edu.

${ }^{1}$ Person-first disability language (e.g., child with autism) and identity-first language (.e.g., autistic child) are used interchangeably throughout this paper, as is the custom in the community I worked with. 
body but created by the social and material conditions that 'dis-able' the full participation of a variety of minds and bodies" [25]. As both a person's body and their environment are constantly in flux, then we can say that a disabled person is not disabled all of the time, but rather, they are disabled by the context (i.e., the world with its objects and the body itself) with which they are trying to engage. As Disability Scholar Titchkosky states, "While we all have bodies—bodies that we act, sense, feel, or move in and through—only some bodies, only some of the time and only in some places, are understood as disabled ones" [65:4]. Access, therefore, is dynamic and ever shifting.

The embodied experience often goes unnoticed and unremarked. In contrast, the disabled embodied experience highlights when a body does not fit into the world creating a remarkable experience for the disabled body [23]. To be "normal" is the expectation that is assumed all other human beings strive for, with every able-body striving to reach perfection and asserting their own normality by comparing their able-bodiness to those who are disabled [44]. The disabled body becomes the oppressed body, when they are denied access (advertently or inadvertently), not only in a social sense, but also in a literal, physical sense. Children who do not conform to the normative embodied experience of play, then, are not invited to the game. This is done both literally and through the crafting of play objects that a disabled body cannot use.

Among other activities, individuals with disabilities use online spaces to socializeempowering themselves to do what they may not be able to in the physical world. Online spaces afford different embodied experiences than do physical spaces. In this ethnographic work, I explore how one community uses the sense of place and the digital embodied experience in a virtual world specifically to give autistic children access to play with their peers. One type of mediated experience is not better (or less mediated) than another. Rather, these experiences, from face-to-face to text to avatars on a computer screen, are diverse kinds of embodied experiences.

The contributions of this work are twofold. First, I demonstrate how various physical and virtual spaces work together to make play possible. Second, I demonstrate these spaces, though some of them are digital, are no more or less "real" than the physical spaces making up a schoolyard or playground.

\section{RELATED WORK}

To ground this work in previous literature, in the following sections, I review social media for individuals with disabilities and scholarly work on virtual worlds and multiplayer digital games. For scholars, our understanding and definitions of disability impact the research conducted in the space of social media and games for individuals with disability. This section covers social media that includes social networking sites, messaging (including email and instant messaging), blogs, forums, as well as virtual worlds and digital games [21]. 


\subsection{Social Media for Individuals with Disabilities}

In the field of HCI, research has mainly focused on the potential benefits and disadvantages of social media, with an implication that these systems are making up for the deficits of the disabled individual user. These works do show the ways in which disabled users may or may not use social media and shed light on some of the reasons why. Burke et al. found through interviews that computer-mediated communication was especially beneficial in helping autistic users initiate communication, but problems occurred once initial contact had been made [10]. Issues of trust, disclosing personal information, and understanding the norms of the social platform made online socialization difficult [10]. Mazurek et al. studied adults with autism and their social media use, finding a majority used the social networking sites for social connections, as opposed to other activities such as reading the news or playing games [42].

Use of social media can empower and increase independence for those with disabilities, but this is not a universal experience. In one literature review, 38 out of 54 articles about social media and virtual world use by individuals with disabilities were focused on inclusion, exclusion, and empowerment [61]. A scholarly focus tends to be on what technology may do for individuals with disabilities, with an emphasis on how the technology acts to support the disabled user. Researchers place much less emphasis on understanding disabled users' relationship with technology-how a user feels about or acts to change their technology. This leads to the "inadvertent propagation of an 'impairment as problem/technology as solution' dynamic" [11]. This is exacerbated when individuals with disabilities are offered children's version of software because it is simpler to use, but can infantilize the user [49]. Social media can empower individual users and create a sense of agency, but as Disability Studies scholars have noted, the tendency to infantilize disabled individuals can have farreaching negative consequences [36]. Young adults and adults with disability need access to age appropriate, yet still accessible technology to enjoy the same privileges as others who can easily access these social media technologies.

Overall, youth are spending more time socializing online, including youth with disabilities [60]. Social media, including blogs and forums, are useful as social supports, providing connection to others in similar situations, and advice for individuals who may not have access to such support in the physical world [61]. Söderström interviewed young adults with varying disabilities about their online and offline social ties and found that patterns of social ties appeared different in youth with disabilities [60]. The offline and online social ties tend to be more blurred together for these users, with less stark contrast between online and offline interactions. This is not surprising given the trend for more youth in general to socialize online [7,34]. Young adults with disabilities may rely on social interactions in virtual spaces more than other young adults; meaning an understanding of how disabled youth interact in these online spaces is becoming increasingly important for scholars, caregivers, and the youth themselves.

\subsection{Virtual Worlds and Multiplayer Digital Games}

While not always included in the category of social media, virtual worlds and multiplayer digital games ${ }^{2}$ do much of the same work as other social media. Virtual worlds are social 
spaces where users have a variety of opportunities to interact with one another. The type of interactions available on the platform can impact how a user engages with that platform, which can be compounded with marginalized users [27]. Communication occurs through both visual and auditory channels, with virtual worlds adding the potential to have more three-dimensional embodied interactions with the user's avatar. For those with disabilities, "virtual worlds offer the possibility of communicating through both text and voice, communication can become less of an obstacle while interacting with others" [62]. Textbased virtual worlds, such as MUDs (e.g., a text-based multi-user dungeon [16]), may be simpler than some other social media with graphical user interfaces-Facebook, for example, has the ability to incorporate photos and video-blurring the lines between these different embodied interactions found in virtual worlds and other social media.

Researchers have conducted limited work on virtual world community members with disabilities. However, scholars have explored both how virtual worlds can allow users to set aside specific spaces (places within the virtual world) for themselves and their community, as well as allow for experimentation with various embodied interactions within these spaces. Second Life is one heavily studied example of having the infrastructure needed to create individual areas for communities. In an ethnography of Second Life, disability was mentioned particularly when it affected the user's ability to use the interface (e.g., typing slowly) and that otherwise, most avatars of disabled users were indistinguishable from ablebodied users [4]. People with disabilities can use virtual worlds to meet new people "on their own terms" where they can be "in control of the situation" [62]. For instance, Second Life has an entire island (i.e., a space set aside within the virtual world) dedicated for users with autism, which is similar to the Autcraft community virtual world [33]. In other work published about the Autcraft community, we found that in creating a safe environment for autistic children, community members redefine what it means to be social in these spaces $[58,59]$. Further, children are active participants in the design, creation, and maintenance of these spaces $[57,58]$.

Virtual worlds also offer an opportunity for those with disabilities to experiment with their avatars, living both real and fantastical experiences online [62]. Users have the choice of whether their avatar has the same disability that they do (e.g., avatar using a wheelchair). Carr found with Deaf users in Second Life that disability is recreated in virtual worlds through discourse and activities [11]. This gives users the chance to escape physical world discrimination and constraints or to play with varying identities, whether those identities have anything to do with disability or not. Individuals with disabilities can experience social interactions virtually that might not be available to them in the physical world [4,62]. Users may want to express their identity within the virtual world as having a disability, which are available in some virtual worlds (e.g., Second Life). While there is the opportunity to embody an able-bodied avatar, likewise, there is the ability to embody an avatar with a disability. Mainstream game developers have begun including disabled avatars (both playable and those characters a player interacts with) in their game content [15].

${ }^{2}$ For the rest of this document I will refer to both virtual worlds and multiplayer digital games as virtual worlds. While there may be some technical distinctions between the two genres, for the purposes of understanding for this work, they are fairly equivalent. 


\section{METHODS}

This paper reports on results from a digital ethnography of an online community that has grown around a Minecraft server known as Autcraft ${ }^{3}$. I collected data through interviews with children and parents, participant observations, directed and non-directed forum discussions, chat logs, and digital artifacts.

\subsection{Setting}

The multiplayer virtual world in our study, Autcraft, is a semi-private server on Minecraft created for children with autism, their families, and allies. Anyone wishing to join must first complete an application. Only those on the "white list" of approved players can access the server. Autcraft currently has more than 7,000 white-listed members with a daily average of approximately 50 players in-world at peak hours of the day and approximately 1,200 individual players logging in each month. While there are no age-restrictions in the community, the content is geared towards members ages 812 years old. The Autcraft community is an inclusive, mixed-ability space.

Minecraft is an open-ended virtual world with no particular goals or play requirements [19,54]. Players can build and create new objects by manipulating blocks in the game. Autcraft is a version of Minecraft that includes modifications and add-ons to the software to allow for the safety of community members [58] and to enhance their socialization [57,59]. In addition to the Minecraft virtual world, the community uses in tandem including YouTube, Twitch, Twitter, Facebook, and a community-maintained website (including an administrator's blog, community forums, member profiles, and an in-browser web messenger).

\subsection{Data Collection}

This work employs virtual ethnographic methods and techniques that have been developed in other studies of virtual world communities [6]. After receiving ethical approval, I gained access to Autcraft via permission of the server's creator for this study and used an avatar labeled as a researcher in-world. Both my presence and purpose were made clear to the community through the Autcraft web-based forum and in the in-world chat. Parents were informed of my presence via a parent message board and the Facebook page of the community. Parents and children were encouraged to voice their concerns and ask questions about the research through all communication platforms used by the community.

From May 2014 to May 2017, I collected approximately 200 hours of immersive in-world observations, including participating in activities on the server, recording chatbased dialogue, and taking extensive field notes on everyday practices of community members and events as they occurred in the virtual world. I also participated in community activities outside the virtual world, including observing discussions in the forums and on the social networking sites. In addition, focus groups were created informally on the forums through forum posts prompts, wherein I asked openended questions of the community. Digital

\footnotetext{
$3_{\text {http://Autcraft.com }}$
} 
artifacts from the various platforms used by the community were also included in the analysis. These data include approximately 5,000 forum threads and 150 blog posts created by players, parents, and administrators.

\subsection{Data Analysis}

I used an iterative, inductive approach where emergent phenomena were identified, named, and categorized following techniques similar to those employed in grounded theory [13]. I used an inductive method of analysis to understand how participants engaged in social play through practice, rather than testing theoretical definitions found in the literature because I was explicitly interested in understanding how the community views and experiences social play. After initial open coding, I used affinity diagramming and axial coding to understand the relationship between, across, and within these codes, the themes of access across the different spaces (i.e., physical, liminal, and virtual) of the Autcraft community emerged. Finally, I reviewed the themes, searching the data for indicators or support for themes, as well as for conflicting data that was not well explained by my original interpretation. This approach was iterative, meaning that I revisited each phase multiple times, as is an established best practice for qualitative data analysis [13]. This paper builds off previously published work in Analog Games [56].

\section{RESULTS}

The Autcraft community actively creates access to social play using a variety of social media and other technology. By looking at the constellation of social media in the Autcraft ecosystem, we gain a more holistic view of how community members are engaging in various social play experiences. These technologies include Minecraft, video via Twitch and YouTube, social networking sites (e.g., Facebook, Twitter), and the Autcraft community website.

Access to the Autcraft community happens through three layers of interconnected environments: physical, liminal, and virtual (See Table 1). The physical space includes computer hardware and the environment in which players access the computer (e.g., bedroom, home office, computer lab in the library). The liminal space includes the installation and configuration of the software, as well as user authentication. Finally, the virtual space includes the various social media. Because of the nature of my ethnography being entirely online, I could only infer some of the ways community members were gaining access, with regard to the physical and liminal spaces, through their own records of these activities within the virtual space. In the following sections, I describe each of these spaces — physical, liminal, and virtual—in the Autcraft community and then follow with an example that impacted each of these spaces.

\subsection{Physical Space}

The physical space is one that includes the physical environment, the computer hardware, and the internet connection needed. How Autcraft community members configure the physical space is one of several points of creating access to social play. In fact, for many individuals with disabilities, this is the first point where access can be limited or denied 
(e.g., mobility impairment prevents someone from interacting with the interface or a visual impairment prevents accessing visual content). Despite the Internet and social media being empowering spaces for disabled individuals, if they cannot gain physical access, they will lose the opportunities afforded to everyone else. Ellcessor states, "digital media cultures take for granted an able-bodied user position" [20]. Assuming one mode of interactivity (e.g., only visual or only auditory) creates disability in other users who are unable to interact in that way $[3,11,20]$.

The physical environment and access to Minecraft poses several different challenges for autistic children — both because they are autistic and because they are children. First, as children, they are often not in control of their physical computing setup-particularly younger children who do not yet have the privilege of their own computers or devices. To further complicate this, families that include individuals with disabilities often live in poverty, further constraining their access to technology [22]. For example, one parent posted in the forums,

"Sadly, it will be just one at a time since we only have one computer semi-capable of handling Minecraft. :( My other computer is from like 2004-ish and I can barely play a game on Facebook with it. But, we will have fun and it will be a lesson in sharing for both of us!"

Many of these children use the family computer, often out in an open space. This means they might not have permission to use the computer whenever they desire, being relegated to times when parents tell them it is okay to play or when their siblings are not using the device. This varies from home to home, but is not questioned when discussed online. A child might say they have to get off now because their older sibling needs to do their homework or that their hour is up. This is met with fond farewells, with everyone understanding why the child must leave. Parents and children must negotiate how and when a child gets access to a computer within each family. Parents often struggle with how much "screen time" to give a child [29], but with autistic children this is complicated by media and experts concerned over the socalled "addiction" to games as a medium $[1,35,41,43]$ and by the child's desire to engage in the virtual world, potentially leading to issues such as temper tantrums and meltdowns [30].

Another important consideration in the physical setup of Minecraft access are the various sensory concerns for those with autism [51]. Autistic children struggle with social play in physical spaces, finding physical touch and eye contact overwhelming in these environments [8]. This includes adjusting the hardware so that it is a more comfortable setup, including dimming or brightening the screen, adjusting the volume of the audio, and even adjusting the brightness of the lights within the physical room. This physical set-up is often mirrored by similar adjustments in the Autcraft virtual world. For example, a child digs a hole in the ground with their avatar to make the screen go black (as opposed to simply turning off the screen). This example shows the analog-digital divide in their play is not as stark or as obvious as one might think - a user moves more seamlessly between and through the two. Creating access happens concurrently across the physical and digital environments. Community members fluidly move from one space to another and simultaneously experience embodiment in all of them. 


\subsection{Liminal Space}

The liminal space between the physical and virtual environments proves a source of contention and access to the Autcraft play space. Liminal space is a concept meaning the transitional space between two states of being or the threshold between two spaces $[24,40]$. The liminal space, in this case, is a distinct space between the physical space and the Autcraft virtual space, a place where critical infrastructure is set up and users maintain the software for the virtual world. This liminal space includes the software, user accounts, and the computer system setup. In the liminal space, one does not necessarily occupy a body as they do in the physical world or virtual world, but nevertheless play still occurs (e.g., overclocking, or running the hardware faster than it is certified to go, or "playing" around with software settings).

Various representations throughout the community (e.g., physical body, avatar, text communication) of an individual's identity are given continuity by stability in their username. A user chooses their own username ${ }^{4}$, which is linked via the Minecraft Mojang account that gives access to an individual's copy of the Minecraft game. These usernames are used across multiple social media platforms. For example, a parent may comment on a Facebook post in the Autcraft community, "My son (Piratescurse) really enjoyed the event today."Referencing the Minecraft username helps to create continuity across the platforms. However, this username can be altered every 30 days; a feature added by Mojang in June 2014. When this feature was originally added, it led to some confusion for Autcraft community members. Some of the members, particularly younger ones, tend to change their names frequently. This is perhaps linked to the more fluid identity of younger individuals [26]. Autcraft administrators implemented new rules (e.g., list old usernames on the public profile) to help maintain the continuity of the community members' names. Through usernames and avatars, Autcraft community members can engage in a variety of embodied experiences across the community's platforms.

The software for Minecraft can be downloaded any number of times to compatible devices with a valid user account login. For some children, this means they have their own account (with their own screen names) and for other children they must share the account with a sibling or parent. Children and parents negotiate and decide where to spend their resources to create access to Minecraft while balancing other priorities in the family-including rules about how much time a child can spend on the computer, how much money a family can afford to spend on access to the game, and the needs of other family members. This becomes more than a simple question of access to game play, but a negotiation over the shared environment and individual values to gain access to the Autcraft community.

After obtaining a license to download and play on the Minecraft software, the next point of access for community members is to join via the application on the website, which requires that an individual have a valid email address linked to a Minecraft account. The application consists of several questions, including "Who has autism in your family?" The wait time for application processing, as of this writing, was approximately two weeks. This wait time is

\footnotetext{
${ }^{4}$ All usernames in this manuscript are pseudonyms.
} 
because a small set of administrators are in control of the whitelist and, as they are volunteers, it takes them time to go through the applications. Setting up access to the server requires multiple steps after the member is accepted into the community. Challenges include ensuring the right version of Minecraft has been selected and installed, dealing with virus protection and firewalls that might prevent the software from accessing the internet, and configuring the operating system to run Minecraft smoothly. These challenges are faced by caregivers and children of the community-those responsible for maintaining the software and hardware needed to run Minecraft and access the other platforms of the Autcraft community.

Through a computer with an internet connection, a child can access the full version of the Minecraft software. While there are mobile editions and console editions of Minecraft, the Autcraft virtual world is only supported through the computer version. However, for children with limited access to a computer, they may also access the chat functionality of the virtual world through third-party mobile applications. These applications log a user into their account and their avatar appears in the virtual world. When using these applications, the user cannot move their avatar or even see the virtual world, except for the text chat (See Figure 1). In this way, they and their avatar embodiment are caught in the liminal space. When a user connects to the virtual world with this application, community members will often signal they are using the mobile application and the server announces in the chat channel:

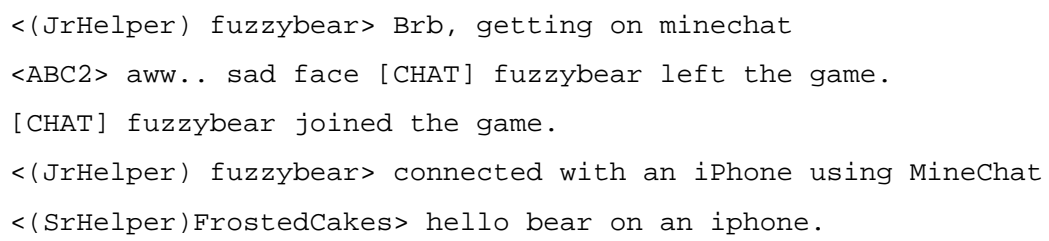

This allows community members to participate in one aspect of the virtual world play, even if they do not have access to the full Minecraft game. Community members learn about these other access options from each other. Following is an excerpt from the chat log in the Autcraft virtual world where community members discuss "minechat":

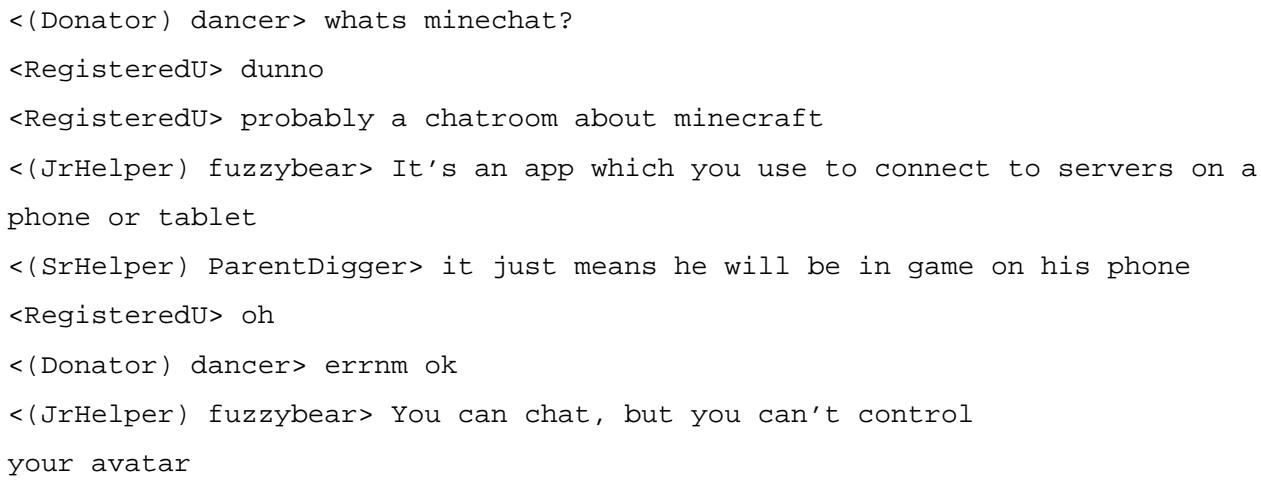


Using these applications to engage in the virtual world show that the child is willing to have some engagement in the virtual world play, or at least the social aspects of this play, rather than none at all. Perhaps given the response from one of the children, "errnm ok," the benefits are not necessarily obvious. A child using the minechat application accepts disabling their avatar to have the ability to engage in some form of social play, rather than have no access at all.

For parents who are able to afford (financially, the energy required to set up, and time) to give their child access, they must also "buy in" to the Autcraft experience-that is, they believe that Autcraft is a valuable place for their children to be spending their time. Much of the parent and child's time is consumed in work, school, and various therapies to help support the autistic child. What little time is left for free play is especially precious. Some parents admit trepidation about allowing too much "screen time" or not understanding the technology or game enough to make informed decisions about their child's access. A whole Autcraft forum is dedicated to helping parents navigate the Autcraft space, while another entire forum is dedicated to solving hardware and software issues. These online forums become almost as important as the virtual world itself. Both children and parents use them to gain the knowledge required to gain entry to the virtual world, which can be intricate and indepth. This knowledge spans hardware and software set-up (including how to add mods and understanding IP addresses) to the social rules needed to navigate the social play within the community.

\subsection{Virtual Space}

The virtual space is the place of digital embodied interaction. This includes the environment of Minecraft inside the Autcraft virtual world, videos, social networking sites, and the Autcraft community website. Throughout the virtual space, avatars represent community members; this is what is seen by other players and interacts in the Autcraft virtual world. The avatar is a blocky humanoid figure that can be edited in appearance through software such as Microsoft's Paint (See Figure 2). Pre-made avatars are available online through searchable databases (e.g., Skindex ${ }^{5}$ ) (See Figure 3). While the shape of the avatar is fixed, players can design with a variety of colors, allowing them to be more than human - from a bear or mermaid to a blue humanoid. Other scholars have pointed to some of the problems with these databases (e.g., underrepresentation of women and people of color), particularly for players of color who are looking to represent themselves in the virtual world [2]. For individuals with disabilities options for representative avatars is limited. All avatars are bipedal and objects, such as wheelchairs, are currently not available. Individuals can also represent themselves through their profile pages and usernames on the website and social media, through their voice or video recordings of their physical world bodies in video streaming and YouTube, and through their own bodies when meeting face-to-face in the physical world.

Following is a description of some of the virtual spaces within the Autcraft community including: the virtual world, video sites, social networking sites, and the community website.

$5_{\text {http://www.minecraftskins.com/ }}$ 
4.3.1 Virtual World.-The Autcraft virtual world is a specific instance of a Minecraft virtual world. Within the Autcraft virtual world, there are different "worlds" that can be accessed via a portal system, similar to "islands" in Second Life. Each of these worlds have different purposes and rules. These separate worlds within the larger Autcraft virtual world allow for players to engage in a variety of activities. These spaces serve as meeting places, areas for building, and a means to go on adventures with other players.

Some places are designed to renew and change every month. From my field notes:

In Autcraft, there is an entire world dedicated to mining raw materials. Mining World is different from other locations in Autcraft because once per month, the world is reset, wiping out everything and making a new world with all new resources. Signs are ubiquitous, warning community members not to build in Mining World because they will lose their creations at the end of the month. However, as the players mine, they also create. Players build functional objects, such as stairs to help reach minerals, but also aesthetically interesting builds (See Figure 4).

These places are designated within the larger Autcraft virtual world, which is nestled in the even larger Autcraft community. These virtual spaces allow for exploration and creativity. They also gives a sense of either permanence or impermanence. In the case of "Mining World," nothing is permanent, as it will be erased at the end of the month. Other spaces, such as player homes, are as permanent as the virtual world is.

4.3.2 Video.-Autcraft community members use two main outlets for creating and publishing video content: Twitch and YouTube. Twitch is a live-streaming video platform used for streaming video game content while the user is playing [37].

Members use this platform to stream live videos of their activities within the Autcraft virtual world. Other Twitch users can search for the Autcraft keyword or find users by username to watch the desired community related content. Live stream videos are meant to be watched in the moment, but are occasionally recorded. Videos recorded and published on YouTube are generally edited before publishing, including title screens denoting the player's username and video title. YouTube videos are shared with other Autcraft members through the community's website. This also included the founder's TED talk about Autcraft, which is widely shared throughout the community [68].

Members follow the example of others they have seen in creating their own videos. For example, one popular Minecraft YouTube channel for children named Stampy ${ }^{6}$ has over 8 million subscribers [67]. Children then emulate these videos, through content such as "Let's Play." "Let's Play" are edited videos of players playing through a video game, giving viewers a first-person experience of playing the game and are popular way to share gaming experiences in many games, including Minecraft [50].

$6_{\text {https://www.youtube.com/channel/UCj5i58mCkAREDqFWlhaQbOw }}$ 
4.3.3 Social Networking Sites.-Autcraft administrators maintain a Twitter account and a Facebook group page, which are used to disseminate community news, post inspirational blogs by members of the community, and keep in touch with community members. Community discussions happen across these sites and are member-driven. For example, announcements are made in multiple places (e.g., Facebook, Twitter, and the website) so that community members can see them on the site they visit more frequently. When media news about the Autcraft community occurs, an administrator posts the link to the article across all the social networking sites. Parents are more likely to respond to posts on Facebook, while more active community members, such as the children, are more likely to respond on the community website. This assures that news reaches many members-even those only indirectly active, such as parents who do not play Minecraft.

4.3.4 Autcraft Community Website.-Autcraft administrators maintain a website that includes a main page with news and blog posts, a status page for the virtual world, forums, member profile pages, and an in-browser web messenger. The website allows members to "friend" each other through profile pages and displays "top forum posters" on the front page for those who have posted the most in the forums (i.e., those who have posted the top number of posts). "Player of the Week" is selected each week by administrators and are players who have stood out to the administration as helpful community members.

In these various places, the ways in which individuals experience virtual embodiment is different. For example, the avatar in the virtual world takes on a much more "physical" embodied experience-interacting in a three-dimensional environment. However, a Facebook profile may not have the same kind of embodied experience. The liminal space in each of these platforms is also different and, in some ways, much less obvious. Again, Facebook requires hardware and an account to access, but the system is supported by a much wider range of hardware and software than Minecraft.

\subsection{The Embodied Experience in the Autcraft Community}

The physical, liminal, and virtual spaces of the Autcraft community are not separate entities, but rather intertwined and intersecting to make up the embodied experience of being in Autcraft. The embodied experience is how an individual engages with their environment through artifacts-implying some kind of bodily experience in those spaces [18]. This is particularly salient when children in the community experience violence.

The Autcraft community explicitly denies access to the play space to one group of individuals - trolls and those who mean harm to the players with autism. This exclusion is controlled mainly through the whitelist of permitted players. The administrators maintain a list of banned usernames that keeps most of the mischief makers at bay. However, one hacking incident led administrators to take more stringent precautions. Hackers had managed to redirect the IP address of the Autcraft server so that when players tried to log in they were sent to another virtual world instead. As reported in an administrator's blog, "Once there, they were encased in a bedrock box from which they could not leave and were told that they were rejects from society, degenerates and that they should kill themselves." When administrators of Autcraft were able to stop the hackers from redirecting Autcraft 
players, the hackers then launched a DDOS (Denial-of-Service) attack on the Autcraft virtual world, which is a means of technically denying access to the virtual world for everyone. As the administrator put it in his blog, the hackers attempted "to make Autcraft unplayable for everyone because if they couldn't tell the children to kill themselves directly, then they'd at least try to take everything away from them that they could."'These attacks eventually caused the Autcraft administrators to change the Autcraft IP address-meaning community members had to understand how to reconfigure their own settings in order not to be locked out along with the hackers. This required quickly educating Autcraft members how to change their settings in order to gain access to the virtual world again. Autcraft administrators had to do this education covertly in order not to alert the hackers to their fix. Administrators made obscure public announcements about "news" that could then be found within the passwordprotected Autcraft website that would hide the new IPaddress from the hackers. This caused some confusion for Autcraft community members, particularly those who are less technologically savvy. Creating access for some inevitably means denying access to others-especially when the goals for one group are in opposition to the goals of another group.

This inclusion of some individuals and the exclusion of others may be an inevitable consequence of trying to create a safe play space for children. How the Autcraft community defines social play — and who are the privileged players - also directly affects those who are excluded. Children with autism are the privileged players within the Autcraft community. While these children are educated when they have misbehaved - explicitly told when they have hurt someone's feelings, for instance-the hackers in the above example are not given that luxury in the same way. The hackers' kind of play runs counter to the ideals of the Autcraft community. In fact, to Autcraft community members, the hackers may not be viewed as players at all because they are involuntarily involving the Autcraft community members in what could be deemed as anti-social play.

In this example, the experience crossed the boundaries of the various spaces-physical, liminal, and virtual — to impact the experiences of community members. As I will discuss further below, this example is particularly salient because despite the acts being "virtual," they are still very real for the children who were victims of them.

\section{DISCUSSION: WHERE IS THE BODY? THE PLACENESS OF THE AUTCRAFT COMMUNITY}

Understanding the infrastructure of the Autcraft community, including the spaces-physical, liminal, and virtual—community members must traverse to engage with each other, as well as the modifications made to Minecraft for the community members' use, allows for a deeper understanding of their embodied experiences in these spaces. Acknowledging that real and unreal experiences can occur in the physical world and in the virtual world means we must also acknowledge the validity of experiences in both spaces. Violent, negative engagement can be painful wherever they may occur. Likewise, positive engagements can lead to meaningful relationships and happy memories no matter where they take place. Whom we choose to let in to these spaces shapes how these interactions unfold. 


\subsection{The Embodiments of the Experience Matter}

The Autcraft community gives their community members many different options for communication and social play through the various platforms discussed above. In doing so, the community accommodates many different user needs across contexts. A parent may not feel comfortable entering and interacting in the Autcraft virtual world, but they are able to leave comments and send messages through Facebook. A young child might not have access to social networking sites, but is able, often with the help of a parent, to navigate their avatar through the Autcraft virtual world and play with others. Being literate is not a requirement for being social in the Autcraft community. While some members choose to access the chat functionality of the virtual world through applications on their phone, other children do not use the chat at all. Instead they rely on their avatars to express themselves and interact with others and the Autcraft virtual world environment.

The embodied experience is different depending on how it is mediated (e.g., face-to-face, text, phone, avatars in the virtual world). However, this does not mean that one is by default better (or less mediated) than the others. Depending on the individual, their "fit" in that mediated experiences may change - not only by type of mediation, but by the context in which that mediation occurs. The "fit" of a mediated experience can directly impact a person's access to social play. Miller and Sinanan argue that all interactions are mediatedincluding those that are face-to-face-even when they do not feel mediated [45]. However, this feeling may be context-dependent and different for each person. In the case of those with autism, some find that physical, bodily sensations are overwhelming or painful and, therefore, seemingly mundane experiences to some, such as eye contact, become unbearable $[12,46]$.

Despite pressure from therapists, parents, educators, and researchers to privilege face-to-face interactions as more "real" than digital interactions, one kind of embodied experience is not necessarily more "real" than another. In fact, what is considered "real" for an individual is highly subjective. Instead of seeing physical and digital in stark contrast to one another, the digital becomes another realm that extends the self and is a place that can contain both the real and the unreal [5]. The premise that real and unreal can occur in both physical and virtual environments is especially salient for those who have lived experiences in both places. To accept that the interactions and encounters that occur in digital spaces are real, is to then accept that negative or hurtful engagements can cause pain when they occur in these spaces. For example, even when the virtual world was entirely based in text, when one rogue user violated other community members' avatars with sexually explicit text, the violation still had a profound impact on the users [17]. Similar violent acts have been recounted across various virtual worlds (e.g., World of Warcraft [48]), as well as other social media platforms [55]. Because this violence is so ubiquitous across social media, the Autcraft community becomes especially important for those who do put value in their own embodied experiences on virtual platforms, such as autistic children. As in face-to-face, physical world interactions, their virtual counterparts also offer positive experiences. Social media has been studied for its ability to foster connections (e.g., intimacy and friendship in virtual worlds $[39,47,52,53,64])$. However, feeling safe is integral to these positive embodied experiences. In turn, feelings of safety help create access to social play. 
The embodied experience is what makes social play possible-embodied experience is the vehicle through which play occurs. The embodied experience gives children the place of play - the tools, other bodies, and boundaries. Whether it is children building a fort out of pillows in the living room or pixels in the Autcraft virtual world, play is made possible by experiences and interactions with the environment.

\subsection{Spaces Become the Autcraft Place}

The physical, liminal, and virtual spaces all intersect to create the place that is Autcraft. Bodies occupy all these spaces simultaneously. How bodies interact in these spaces-how engagement is mediated - depends on the context. A person could play with words at home at the dinner table, play a game of tag in the school playground, play with computer settings on a laptop in the library, or hide and seek in a Minecraft virtual world. If "space is the opportunity," then Autcraft becomes the "understood reality" [28]. The constellation of platforms used by Autcraft community members- from social networking sites, streaming video, specialized computer hardware to, lest we forget, Minecraft—converge into one giant space, or maybe many smaller spaces linked together with bridges. Through careful setting out of social norms and rules by the administrators, the Autcraft community takes the "opportunity" of this space and creates a sense of place, or, as at least one Autcraft member has called it, "home."

\subsection{What is "real"?}

The common complaint about computer games and children's online activity is when they are engaging online ("on screens"), they are not engaging with "the real world." I am deliberate when labeling the spaces used in the Autcraft community-physical, liminal, and virtual. All of these spaces can hold aspects of the real and unreal [5]. The Autcraft community—-through their play—demonstrate this. One of the aspects of play is the suspension of belief by players. In play, we can accept both the real and unreal side-byside. As Sutton-Smith stated, "Children's play fantasies are not meant only to replicate the world...; they are meant to fabricate another world that lives alongside the first one and carries on its own kind of life, a life often much more emotionally vivid than mundane reality" [63:158]. Play includes shared imagining and creation of shared fictitious worlds; "...the relationships between the members of the community of players are relationships of collaboration in creating a fictive world" [38:56]. Minecraft, as a platform, makes possible the realization of the children's fantasy worlds. Just as a block tower in a school might become a knight's castle, Minecraft gives the children the tools to make their own fantasy castles and act out their play with each other through their avatars. But what is real here and what is not? A block tower, many would argue is real in its physicality, but not necessarily really a knight's castle. Is a digital block tower in Minecraft not real simply because of its digital-ness? The two should be treated similarly. The children of Autcraft play with their digital towers with more gusto than they might in the physical world - where they are made disabled by the chosen environment.

The outcomes of play in Autcraft have yet to be measured statistically. It is unknown if children from the Autcraft community "transfer" their social "skills" into other aspects of their lives. This does not need to be the only end goal of the Autcraft community, however. 
The Autcraft community, through their constellation of platforms, gives members a place to play. Having access to play for play's sake is an important means in itself.

\subsection{Topos-Mediated Ludic Sociality and the Able Body}

Place either aids or hinders access to play. As in the example of the playground, a place is not simply a demarcated area of space. Place is imbued with cultural meaning and social cues-that is how a space becomes a place. In a playground, the place is full of cues for play - a playground invites play. In other words, a playground is a playful place, meant to be full of play. Topos is "common ground" or "place" in Ancient Greek. The playground-in this case, a Minecraft virtual world - becomes the topos or "common ground" where ludic sociality occurs. In this section, I discuss how place or topos becomes the mediator for ludic sociality.

All communication is culturally mediated [45], but the impact of technologies such as smart phones and virtual worlds is more noticeable by society - both because of what is seen and not seen. Technologies are "sometimes unsettling largely because they make us aware and newly self-conscious about those taken-for-granted frames around direct face-to-face encounters," making us see how sociality is mediated [31]. But technology is also disconcerting to people because of what they cannot see. For example, watching children in virtual worlds is more challenging especially for caregivers who do not understand the technology. Children who prefer these digital engagements may be seen as antisocial or casting-off the "real" world [66]. However, there is more happening in these virtual worlds than is first apparent. Consider the disabled embodied experience- those who are considered disabled are such because the environment does not support a particular body $[23,25]$. The children (and adults) of the Autcraft community prefer-and are more comfortable with-interactions that are mediated via text or digital avatar. They are denied access to - that is, they are made disabled — when forced to use the mediations that do not fit. I look at play, and the access to play, as a way to understand the importance of sociality to autistic children, but also to show how we can leverage technology to create more inclusive social experiences through ludic sociality.

If "play is the work of children," [14] then what is the product of their work? Ludic sociality can be understood as the ways in which play mediates being social. All communication is culturally mediated [45] and "culture arises in the form of play, that it is played from the very beginning" [32:46]. However, neither play nor sociality require one another. One can play without others, and one can be social without play. Play can be one of the layers of mediation that helps support sociality and create community.

The children express their sociality through play. Play could occur because the children had access to play in a context in which they "fit." When educators, therapists, parents, and researchers privilege face-to-face interactions, they are, in effect, creating disability in children who cannot or will not play in that medium. In the Autcraft community, members can recreate the playground in an environment that is more comfortable for them. Community members leverage technology to create a playground where the body is most able to play. And, having done this, autistic children engage in social play of which people thought them incapable. 
As I noted above, the Autcraft community has used a constellation of platforms to enable ludic sociality for autistic children. Here the Autcraft community is using the constellation of technological platforms to help create the sense of place. These platforms cross into physical, liminal, and virtual spaces, working together to create access to play.

Toposmediated ludic sociality is how the place mediates and creates access to ludic sociality. Here the Autcraft community have leveraged virtual worlds to their advantage—in essence, transforming the disabled embodied experience into an enabled one.

\section{CONCLUSION}

I discussed the embodied experience of those with disabilities, especially regarding social media. I then described how these embodied interactions occur throughout the Autcraft community including the physical, liminal, and virtual spaces Autcraft community members live in. Computer-mediated experiences for these community members is preferable to physical-world, face-to-face interactions. How someone interacts with others is meaningful, regardless whether that interaction is online or offline. The Autcraft community has defied the conventions set out by many educators, parents, researchers, and therapists by creating a space that privileges digital engagements over physicalworld ones. In doing so, they have made a playground that is more comfortable for many autistic children. Ultimately, it the placeness of Autcraft that gives children the options of embodied experiences they need to access social play.

Children need a place to play. There are two things to consider: creating a sense of place and allowing for both the real and unreal in these places. Therapists, educators, parents, and researchers tend to privilege the physical realm over all others for mediating sociality. Members of the Autcraft community turn this notion on its head, instead privileging virtual interactions over physical ones. But further still, I have shown how all these spacesphysical, liminal, and virtual-must work together to make play possible. The spaces the Autcraft community uses make the place that is Autcraft possible. Second, these spaces, though some of them are digital, are no more or less "real" than the physical spaces making up a schoolyard or playground.

\section{ACKNOWLEDGMENTS}

I thank the members of Autcraft for the warm welcome to their community. I would like to thank Chris Wolf, LouAnne Boyd, and Oliver Haimson and other members of LUCI for their feedback on various iterations of this work. Thank you to Severn Ringland and Kyle Lee for feedback on this work. Special thanks to: Gillian Hayes, Tom Boellstorff, Mimi Ito, and Aaron Trammell. I would also like to thank Robert and Barbara Kleist for their support, as well as the ARCS Foundation. This work is supported by the National Institute of Mental Health (T32MH115882). The content is solely the responsibility of the authors and does not necessarily represent the official views of the NIH. This work is covered by human subjects protocol \#2014-1079 at the University of California, Irvine.

\section{REFERENCES}

[1]. Alper Meryl. 2014 Digital Youth with Disabilities MIT Press, Cambridge, MA.

[2]. Ames Morgan G. and Burrell Jenna. 2017 'Connected Learning' and the Equity Agenda: A Microsociology of Minecraft Play. In CSCW 2017 Retrieved February 1, 2017 from http:// morganya.org/research/ames-cscw17-mine-craft.pdf 
[3]. Baldwin Mark S., Hayes Gillian R., Haimson Oliver L., Mankoff Jennifer, and Hudson Scott E.. 2017 The Tangible Desktop: A Multimodal Approach to Nonvisual Computing. ACM Transactions on Accessible Computing 10, 3: 1-28. 10.1145/3075222

[4]. Boellstorff Tom. 2010 Coming of Age in Second Life: An Anthropologist Explores the Virtually Human Princeton University Press.

[5]. Boellstorff Tom. 2016 For Whom the Ontology Turns: Theorizing the Digital Real. Current Anthropology 57, 4: 387-407. 10.1086/687362

[6]. Boellstorff Tom, Nardi Bonnie, Pearce Celia, and Taylor TL. 2012 Ethnography and Virtual Worlds: A Handbook of Method Princeton University Press.

[7]. boyd danah. 2014 It's Complicated: the social lives of networked teens Yale University Press.

[8]. Boyd LouAnne E., Ringland Kathryn E., Haimson Oliver L., Fernandez Helen, Bistarkey Maria, and Hayes Gillian R.. 2015 Evaluating a Collaborative iPad Game's Impact on Social Relationships for Children with Autism Spectrum Disorder. ACM Transactions on Accessible Computing 7, 1: 1-18. 10.1145/2751564

[9]. Burke Anne and Marsh Jackie (eds.). 2013 Children's Virtual Play Worlds: Culture, Learning, and Participation Peter Lang Publishing, Inc., New York.

[10]. Burke Moira, Kraut Robert, and Williams Diane. 2010 Social use of computer-mediated communication by adults on the autism spectrum. In Proceedings of the 2010 ACM conference on Computer supported cooperative work, 425-434. Retrieved January 12, 2014 from http:// dl.acm.org/citation.cfm?id=1718991

[11]. Carr Diane. 2011 Constructing Disability in Online Worlds: Conceptualizing Disability in Online Research. In Reinventing Ourselves: Contemporary Concepts of Identity in Virtual Worlds, Peachey Anna and Childs Mark (eds.). Springer London, London, 177-190. 10.1007/978-0-85729-361-9

[12]. Cesaroni Laura and Garber Malcolm. 1991 Exploring the experience of autism through firsthand accounts. Journal of Autism and Developmental Disorders 21, 3: 303-313. [PubMed: 1938776]

[13]. Charmaz Kathy. 2006 Constructing Grounded Theory: A Practical Guide to Qualitative Analysis Sage Publications Ltd.

[14]. Cross Gary. 1997 Kids' Stuff: Toys and the Changing World of American Childhood Harvard University Press.

[15]. Amanda LL Cullen Kathryn E. Ringland, and Wolf Christine T.. 2018 A Future Worth Fighting For: Representation of Disability in Overwatch. First Person Scholar

[16]. Dibbell Julian. 1998 My Tiny Life: Crime and Passion in a Virtual World Henry Holt and Company, Inc., New York.

[17]. Dibbell Julian. 1998 A Rape in Cyberspace: Or a Tinysociety, and how to make one. In Tinylife: crime and passion in a virtual world Henry Holt and Company, Inc., New York, N.Y., 11-30.

[18]. Dourish Paul. 2001 Where the Action Is: The Foundations of Embodied Interaction MIT Press, Cambridge, MA.

[19]. Duncan Sean C.. 2011 Minecraft, beyond construction and survival. Well Played: a journal on video games, value and meaning 1, 1: 1-22.

[20]. Ellcessor Elizabeth. 2016 Restricted Access: Media, Disability, and the Politics of Participation NYU Press.

[21]. Fuchs Christian. 2017 What Are Social Media and Big Data? In Social media: a critical introduction (2nd edition). SAGE Publications, Thousand Oaks, CA, 33-64.

[22]. Fujiura Glenn T. and Yamaki Kiyoshi. 2000 Trends in demography of childhood poverty and disability. Exceptional children 66, 2: 187-199.

[23]. Garland-Thomson Rosemarie. 2011 Misfits: A feminist materialist disability concept. Hypatia 26, 3: 591-609.

[24]. Gennep Arnold van. 1960 The Rites of Passage University of Chicago Press.

[25]. Ginsburg Faye and Rapp Rayna. 2013 Disability Worlds. Annual Review of Anthropology 42, 1: 53-68. 10.1146/annurev-anthro-092412-155502 
[26]. Grotevant Harold D. and Cooper Catherine R.. 1985 Patterns of Interaction in Family Relationships and the Development of Identity Exploration in Adolescence. Child Development 56, 2: 415 10.2307/1129730 [PubMed: 3987416]

[27]. Haimson Oliver L.. 2018 The Social Complexities of Transgender Identity Disclosure on Social Media ProQuest, University of California Irvine.

[28]. Harrison Steve and Dourish Paul. 1996 Re-place-ing space: the roles of place and space in collaborative systems. In Proceedings of the 1996 ACM conference on Computer supported cooperative work, 67-76. Retrieved April 28, 2015 from http://dl.acm.org/citation.cfm? $\mathrm{id}=240193$

[29]. Hiniker Alexis, Schoenebeck Sarita Y., and Kientz Julie A. 2016 Not at the Dinner Table: Parents- and Children-s Perspectives on Family Technology Rules 1374-1387. $10.1145 / 2818048.2819940$

[30]. Hiniker Alexis, Suh Hyewon, Cao Sabina, and Kientz Julie A.. 2016 Screen Time Tantrums: How Families Manage Screen Media Experiences for Toddlers and Preschoolers 648-660. $10.1145 / 2858036.2858278$

[31]. Horst Heather A. and Miller Daniel (eds.). 2012 Digital Anthropology Bloomsbury Academic.

[32]. Huizinga Johan. 1949 Homo Ludens: A Study of the Play-Element in Culture Routledge and Kegan Paul, London; Boston, Mass.

[33]. Irani Lilly C., Hayes Gillian R., and Dourish Paul. 2008 Situated practices of looking: visual practice in an online world. In Proceedings of the 2008 ACM conference on Computer supported cooperative work, 187-196. Retrieved January 31, 2014 from http://dl.acm.org/citation.cfm? id $=1460592$

[34]. Mizuko Itō Sonja Baumer, Bittanti Matteo, danah boyd, Cody Rachel, Herr-Stephenson Becky, Horst Heather A., Lange Patricia G, Mahendran Dilan, Martinez Katynka Z., Pascoe CJ, Perkel Dan, Robinson Laura, Sims Christo, and Tripp Lisa. 2010 Hanging Out, Messing Around, and Geeking Out: Kids Living and Learning with New Media MIT Press, Cambridge, MA.

[35]. Kardaras Nicholas. 2016 Glow Kids: How Screen Addiction is Hijacking Our Kids and How to Break the Trance St. Martin's Press.

[36]. Luborsky Mark R.. 1994 The cultural adversity of physical disability: Erosion of full adult personhood. Journal of Aging Studies 8, 3: 239-253. [PubMed: 25360062]

[37]. MacCallum-Stewart Esther. 2014 "Someone off the YouTubez": The Yogscast as Fan Producers. In Understanding Minecraft: Essays on Play, Community, and Possibilities, Garrelts Nate (ed.). McFarland \& Company, Inc., Jefferson, NC, 148-159.

[38]. Marjanovic-Shane Ana. 2010 From Yes and No to Me and You: A Playful Change in Relationships and Meanings. In Vygotsky and Creativity: A Cultural-historical Approach to Play, Meaning Making, and the Arts, Cathrene Connery M, John-Steiner Vera P. and MarjanovicShane Ana (eds.). Peter Lang Publishing, Inc., New York, 41-59.

[39]. Marsh Jackie. 2013 Breaking the Ice: Play, Friendships, and Online Identities in Young Children's Use of Virtual Worlds. In Children's Virtual Play Worlds: Culture, Learning, and Participation, Burke Anne and Marsh Jackie (eds.). Peter Lang Publishing, Inc., New York, 5978.

[40]. Marae Martinez Nicola. 2011 Liminal Phases of Avatar Identity Formation in Virtual World Communities. In Reinventing Ourselves: Contemporary Concepts of Identity in Worlds Virtual, Peachey Anna and Childs Mark (eds.). Springer London, London, 59-81. 10.1007/978-0-85729-361-9

[41]. Mazurek MO and Engelhardt CR. 2013 Video Game Use in Boys With Autism Spectrum Disorder, ADHD, or Typical Development. PEDIATRICS 132, 2: 260-266. 10.1542/peds. 2012-3956 [PubMed: 23897915]

[42]. Mazurek Micah O.. 2013 Social media use among adults with autism spectrum disorders. Computers in Human Behavior 29, 4: 1709-1714. 10.1016/j.chb.2013.02.004

[43]. Mazurek Micah O. and Engelhardt Christopher R.. 2013 Video game use and problem behaviors in boys with autism spectrum disorders. Research in Autism Spectrum Disorders 7, 2: 316-324. 10.1016/j.rasd.2012.09.008 
[44]. McRuer Robert. 2010 Compulsory Able-Bodiness and Queer/Disabled Existence. In The Disability Studies Reader (3rd ed.), Davis Lennard J. (ed.). Routledge, 369-378.

[45]. Miller Daniel and Sinanan Jolynna. 2014 Webcam Polity, Cambridge.

[46]. Eve Müller Adriana Schuler, and Yates Gregory B.. 2008 Social challenges and supports from the perspective of individuals with Asperger syndrome and other autism spectrum disabilities. Autism 12, 2: 173-190. 10.1177/1362361307086664 [PubMed: 18308766]

[47]. Munn Nicholas John. 2012 The reality of friendship within immersive virtual worlds. Ethics and Information Technology 14, 1: 1-10. 10.1007/s10676-011-92746

[48]. Nardi Bonnie. 2010 My Life as a Night Elf Priest An Anthropological Account of World of Warcraft University of Michigan Press.

[49]. Näslund Rebecka and Gardelli Åsa. 2013 'I know, I can, I will try': youths and adults with intellectual disabilities in Sweden using information and communication technology in their everyday life. Disability \& Society 28, 1: 28-40. 10.1080/09687599.2012.695528

[50]. Nguyen Josef. 2016 Performing as video game players in Let's Plays. Transformative Works and Cultures 22, 0 Retrieved September 20, 2016 from http://journal.transformativeworks.org/ index.php/twc/article/view/698

[51]. Ochs Elinor and Solomon Olga. 2010 Autistic Sociality. Ethos 38, 1: 69-92. 10.1111/j. 1548-1352.2009.01082.x

[52]. Pace Tyler, Bardzell Shaowen, and Bardzell Jeffrey. 2010 The rogue in the lovely black dress: intimacy in world of warcraft. In Proceedings of the SIGCHI Conference on Human Factors in Computing Systems, 233-242. Retrieved November 11, 2014 from http://dl.acm.org/ citation.cfm?id=1753361

[53]. Pearce Celia and Artemesia. 2009 Communities of Play: Emergent Cultures in Multiplayer Games and Virtual Worlds MIT Press.

[54]. "Notch” Persson Markus. 2011 Minecraft Mojang, Stockholm, Sweden.

[55]. Phillips Whitney. 2011 LOLing at tragedy: Facebook trolls, memorial pages and resistance to grief online. First Monday 16, 12 Retrieved January 22, 2018 from http://firstmonday.org/ojs/ index.php/fm/article/view/3168

[56]. Ringland Kathryn E.. 2017 Who Has Access? Making Accessible Play Spaces in Minecraft for Children with Autism. Analog Games Studies 4, 3.

[57]. Ringland Kathryn E., Wolf Christine T., Boyd LouAnne E., Baldwin Mark, and Hayes Gillian R.. 2016 Would You Be Mine: Appropriating Minecraft as an Assistive Technology for Youth with Autism. In ASSETS 2016

[58]. Ringland Kathryn E., Wolf Christine T., Dombrowski Lynn, and Hayes Gillian R.. 2015 Making "Safe": CommunityCentered Practices in a Virtual World Dedicated to Children with Autism. In CSCW 2015

[59]. Ringland Kathryn E., Wolf Christine T., Faucett Heather, Dombrowski Lynn, and Hayes Gillian R.. 2016 "Will I always be not social?": Re-Conceptualizing Sociality in the Context of a Minecraft Community for Autism. In CHI 2016

[60]. Söderström Sylvia. 2009 Offline social ties and online use of computers: A study of disabled youth and their use of ICT advances. New media \& society 11, 5: 709-727.

[61]. Stendal Karen. 2012 How do People with Disability Use and Experience Virtual Worlds and ICT: A Literature Review. Journal of Virtual World Research 5, 1.

[62]. Stendal Karen, Balandin Susan, and Molka-Danielsen Judith. 2011 Virtual worlds: A new opportunity for people with lifelong disability? Journal of Intellectual and Developmental Disability 36, 1: 80-83. 10.3109/13668250.2011.526597 [PubMed: 21070118]

[63]. Sutton-Smith Brian. 1997 The Ambiguity of Play Harvard University Press, Cambridge, MA.

[64]. Taylor TL. 2006 Play Between Worlds MIT Press, Cambridge, MA.

[65]. Titchkosky Tanya. 2011 The Question of Access: Disability, Space, Meaning University of Toronto Press, Toronto, Ontario, Canada.

[66]. Turkle Sherry. 2012 Alone Together: Why We Expect More from Technology and Less from Each Other Basic Books. 
[67]. 2016 The 12 Best Kid-Friendly Minecraft Channels on YouTube Retrieved February 1, 2017 from https://www.commonsensemedia.org/blog/the-12-best-kidfriendly-minecraft-channels-onyoutube

[68]. 2016 How I use Minecraft to help kids with autism Retrieved from https://www.ted.com/talks/ stuart_duncan_how_i_use_minecraft_to_help_kids_with_autism?language=en 
CCS CONCEPTS

- $\quad$ Human-centered computing $\rightarrow$ Accessibility; Accessibility theory, concepts and paradigms 


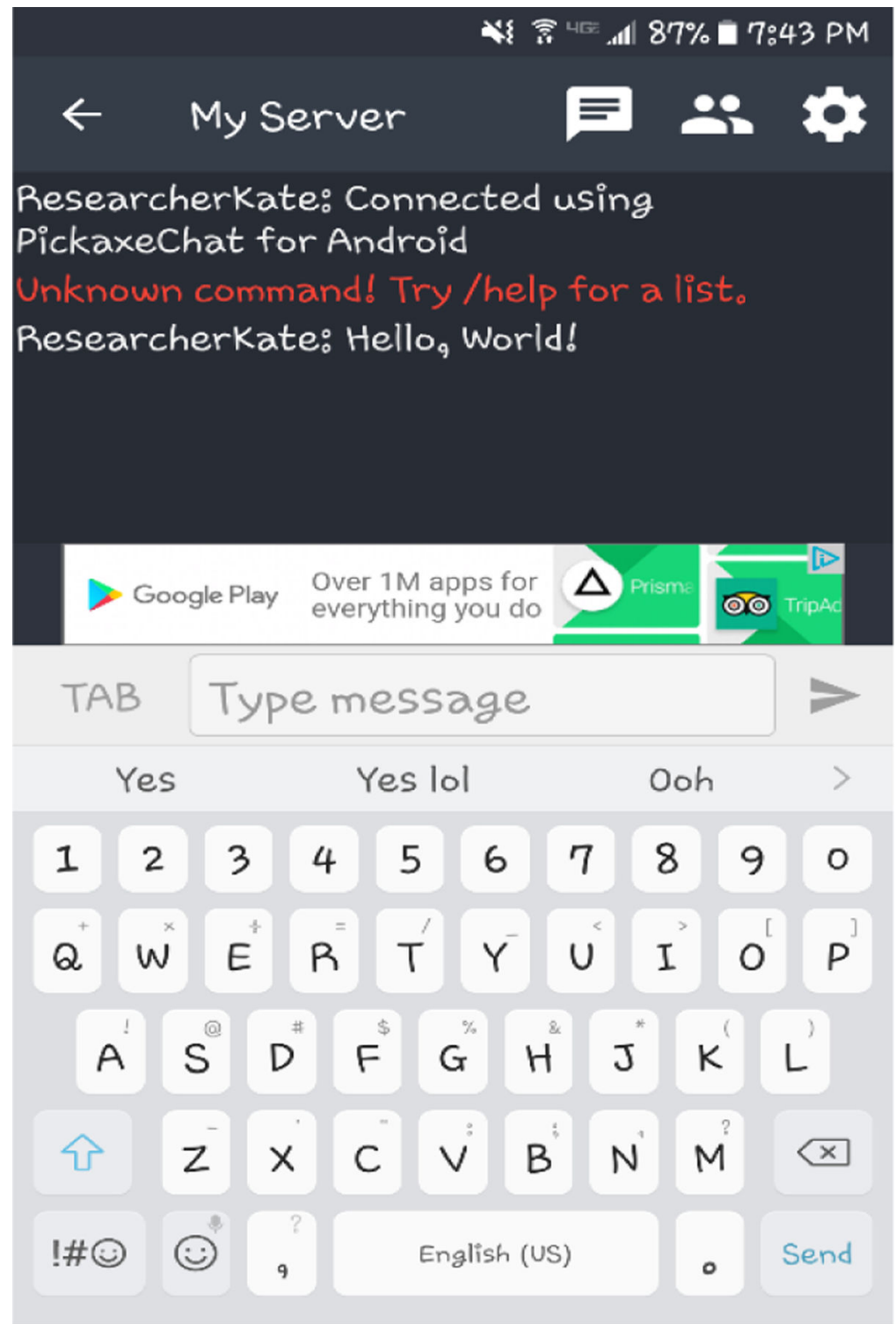

Figure 1.

A screen shot of the chat app for mobile devices. 


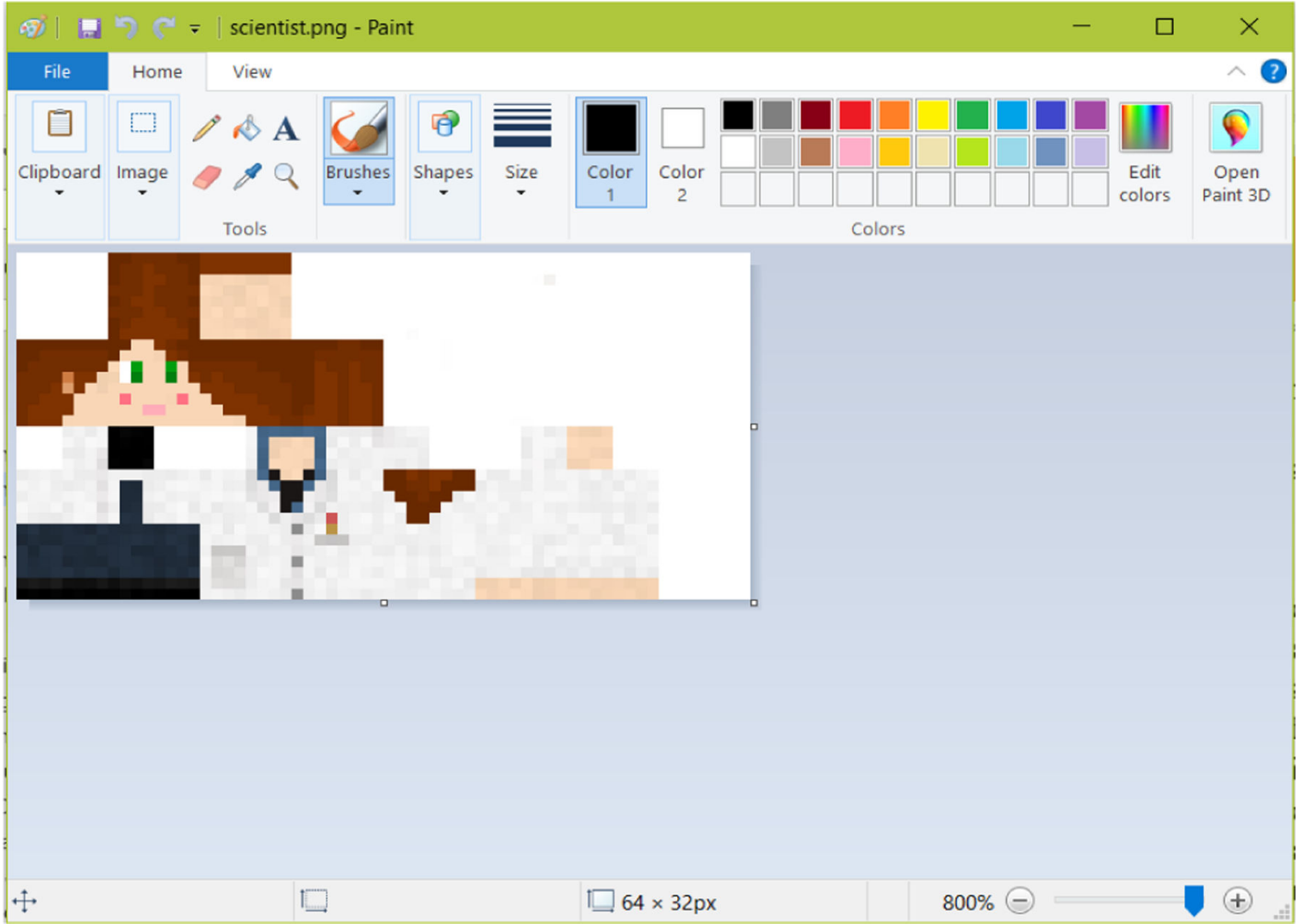

Figure 2.

Editing my Minecraft avatar in Microsoft's Paint program. 

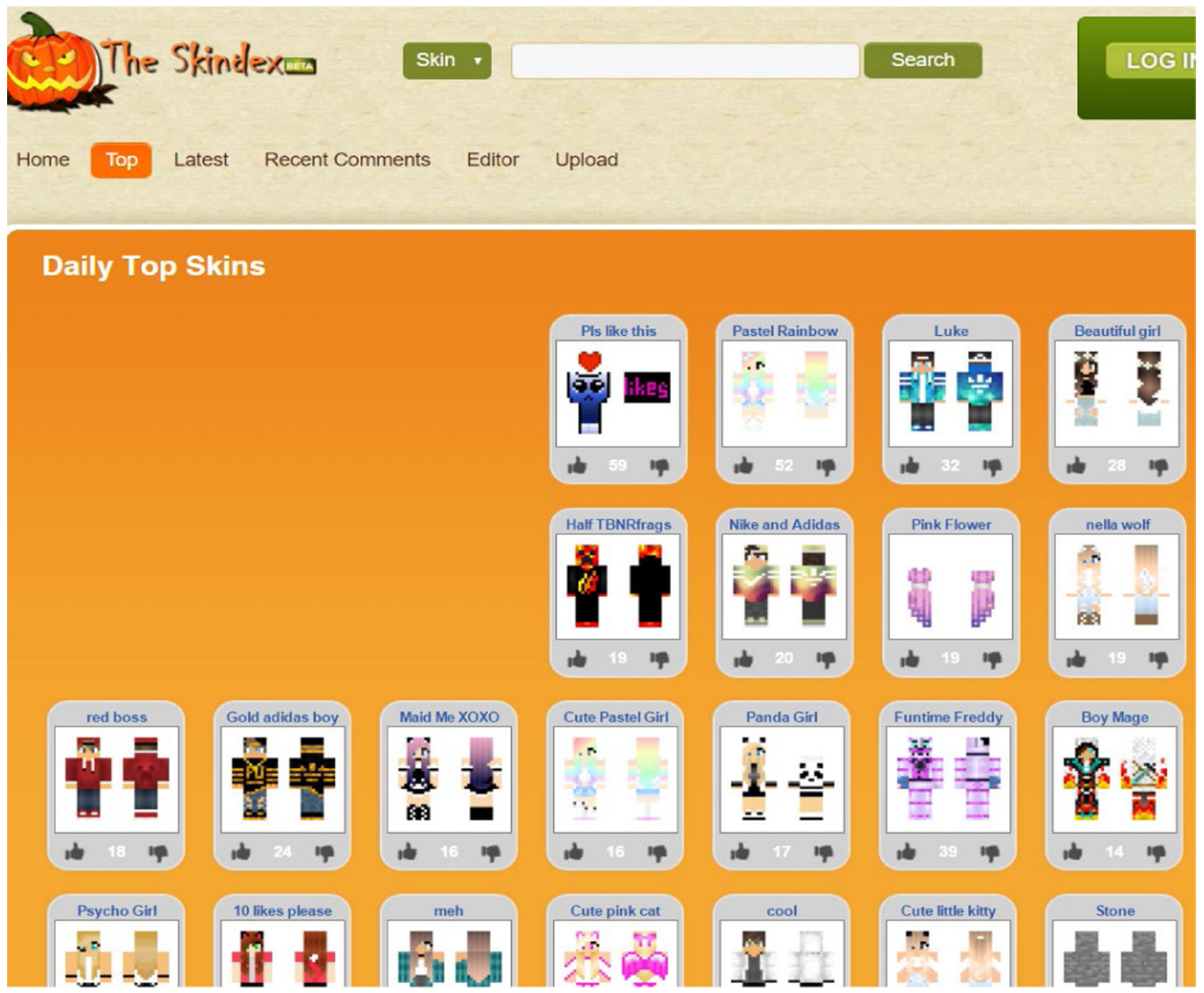

Figure 3.

A screenshot of the Skindex website displaying the daily top skins available for download. 


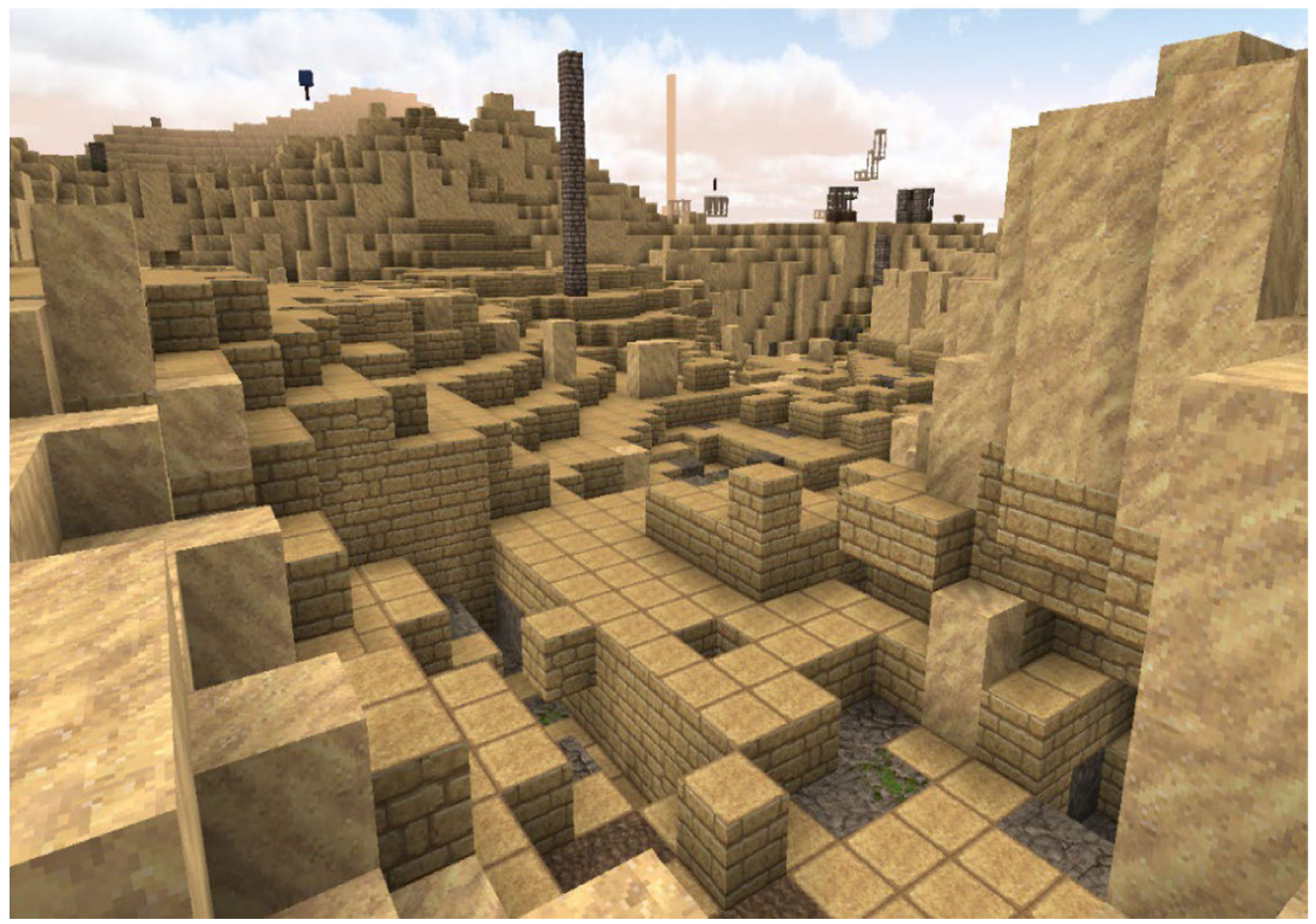

Figure 4.

Mining World with tower structures in the distance. 
Table 1.

The different "layers" of environments that affect access to play in the Autcraft community.

\begin{tabular}{|l|l|}
\hline Physical & Computer hardware physical space \\
\hline Liminal & Software configuration Authentication and Subscriptions \\
\hline Virtual & Minecraft Social Networking Sites (e.g. Facebook Twitter), Video (e.g., YouTube, Twitch), Autcraft community website \\
\hline
\end{tabular}

\begin{tabular}{|l|l|}
\hline Physical & Computer hardware physical space \\
\hline
\end{tabular}

Liminal inecraft Social Networking Sites (e.g. Facebook Twitter), Video (e.g., YouTube, Twitch), Autcraft community website 